\title{
ANÁLISIS DEL COMPORTAMIENTO REOLÓGICO Y TERMO- HIGROMECÁNICO EN PROBETAS DE TABLEROS TENSADOS DE MADERA PARA PUENTES A CONDICIONES AMBIENTALES VARIABLES
}

\section{ANALYSIS OF RHEOLOGICAL AND THERMO-HYGRO-MECHANICAL BEHAVIOUR OF STRESS-LAMINATED TIMBER BRIDGE DECK IN VARIABLE ENVIRONMENTAL CONDITIONS}

\author{
Macarena Figueroa ${ }^{1}$, Cecilia Bustos ${ }^{2 \wedge}$, Peter Dechent ${ }^{3}$, Laura Reyes ${ }^{4}$, \\ Alain Cloutier ${ }^{5}$, Mario Giuliano ${ }^{3}$.
}

\begin{abstract}
RESUMEN
La madera presenta un comportamiento termo-higromecánico cuando se somete a la acción conjunta de variaciones de temperatura, humedad relativa y carga mecánica. Los tableros tensados de madera presentan pérdidas de fuerza en la barra de tensado debido principalmente a la relajación de la tensión y a la contracción de la madera, aumentando con los cambios de humedad y temperatura de ésta. El objetivo de este estudio fue estudiar el comportamiento reológico de tableros tensados de madera de pino radiata (Pinus radiata D. Don) sometidos a cambios de temperatura y humedad relativa. Un total de 16 probetas fueron previamente tratadas con diferentes tratamientos de preservación, las cuales fueron sometidas a cuatro ciclos de temperatura y humedad relativa en una cámara de clima. La deformación de las probetas y la tensión de las barras de acero fueron medidas. Los resultados mostraron que la variación de la temperatura y de la humedad tiene una fuerte influencia en la variación de la tensión de las barras tensoras. Los retensados minimizaron la pérdida de tensión de las barras. Además, no se observaron evidencias de fluencia termo-higromecánica ni relajación de la tensión de las muestras de puentes tensados en este estudio.
\end{abstract}

Palabras clave: Puentes tensados, tensión en las barras, tratamientos de preservación, Pinus radiata D.Don.

\footnotetext{
ABSTRACT

Wood material has a thermo-hygromechanical behaviour because its deformation depends on the combined action of temperature, relative humidity and mechanical load variations. Stresslaminated timber bridge deck show a reduction of stress in the tension bars mainly due to the relaxation and shrinkage of wood, increasing with moisture content and temperature changes within the wood. The aim of this research was to study the rheological behavior of stress-laminated timber bridge deck samples under temperature and relative humidity variations. A total of 16 samples were previously treated with four types of preservative treatments, which were subjected to four cycles of temperature and relative humidity in a climate chamber. Wood samples deformation and stress in the steel bars were measured. The results showed a significant stress reduction in the steel bar during

${ }^{1}$ Estudiante de Magíster en Ciencia y Tecnología de la Madera, Centro de Biomateriales e Ingeniería (CBI), Universidad del Bío-Bío. Concepción, Chile.

${ }^{2}$ Profesor Asociado, Ph.D., Centro de Biomateriales y Nanotecnología (CBN), Departamento de Ingeniería en Maderas, Universidad del BíoBío. Concepción, Chile.

${ }^{3}$ Profesor Asociado, Departamento de Ingeniería Civil. Universidad de Concepción. Concepción, Chile.

${ }^{4}$ Profesor Asociado, MSc., Departamento de Ingeniería en Maderas, Universidad del Bío-Bío. Concepción, Chile.

${ }^{5}$ Profesor Ttitular, Ph.D., Centre de Recherche sur le Bois, Université Laval. Québec, Canada.

Autor para correspondencia: ^cbustos@ubiobio.cl

Recibido: 01.05.2011 Aceptado: 10.04.2012
} 
the summer season and a significant increase during the winter season. The results also showed significant differences between the deformations of control samples compared to the preservative treated samples for each cycle. However, for interlaminar stress, differences were significant only in the winter season. Moreover, for both deformation and interlaminar stress, no significant differences between cycles three and four were found. Therefore there is no evidence of thermo-hygromechanical creep or relaxation of wood in the conditions considered in this study.

Keywords: Stress-laminated timber bridge deck, bar stress, preservation treatments, Pinus radiata D.Don.

\section{INTRODUCCIÓN}

Los puentes de tableros tensados de madera fueron originalmente desarrollados en Ontario, Canadá en 1976 y consisten en tablones dispuestos de canto, los que en conjunto son comprimidos en sentido transversal por medio de barras de acero de alta resistencia (Ugalde 2004, Dyken 2005, Gilún y Meronk 2006, Guiliano et al. 2011). En este tipo de puentes, los tablones de madera se encuentran cargados transversalmente durante toda su vida útil y pueden sufrir, en el tiempo, deformaciones por fluencia, produciéndose un acortamiento elástico de la barra de acero y la consecuente disminución de la fuerza de compresión del tablero (Huaiquilaf 2004). Además, la influencia de las variaciones de temperatura y humedad relativa producen en la madera cambios volumétricos que también afectan el tensado de las barras (Gallegos 2009). La madera presenta deformación a lo largo del tiempo cuando ésta es sometida a una carga permanente producto del comportamiento reológico, fluencia y relajación, característico de los materiales viscoelásticos. La pérdida de tensión en las barras puede ser controlada con el número de veces que el tablero es retensado, pudiendo limitar esta pérdida a un máximo de 60\%, cuando el tablero es retensado una segunda vez dentro de un periodo de tiempo determinado (Ritter 1990). Estos comportamientos desarrollados en estructuras de madera, obedecen principalmente a la acción mixta de la temperatura, de la humedad relativa y de la carga mecánica que actúan en el material. A este tipo de comportamiento se le denomina comportamiento termohigromecánico (Navi y Heger 2005).

Varios autores han estudiado el efecto de humedad y temperatura en puentes de tableros tensados de madera (Ritter et al. 1996, Wacker et al. 1996, Kainz et al. 2001). En un estudio llevado a cabo por Kainz et al. (2001), se mostró que los tableros de madera tensados presentan un buen desempeño a temperaturas extremadamente bajas mientras su contenido de humedad sea inferior a $19 \%$. Cuando la humedad se encuentra por sobre el punto de saturación de la fibra (PSF), la pérdida de fuerza en la barra aumenta considerablemente. Del mismo modo, Wacker et al. (1996) establecieron que a medida que disminuye la temperatura, la pérdida de fuerza en la barra aumenta, provocando una acelerada contracción en la madera a temperaturas que se encuentran por debajo de $0{ }^{\circ} \mathrm{C}$ y a un contenido de humedad por sobre el punto de saturación de las fibras. Un ejemplo de la variación del tensado en puentes lo constituye el puente Hoffman Run de Estados Unidos, compuesto de un tablero tensado de madera tratada con creosota, el que presentó una pérdida de fuerza en las barras, atribuida principalmente, a la relajación del tensado en las laminaciones del tablero como resultado del alto contenido de humedad presente en la madera, Ritter et al. (1996).

\section{Fluencia y Relajación en la madera}

La deformación por fluencia representa la evolución de la respuesta mecánica de una estructura en el transcurso del tiempo bajo la acción de una carga constante (Bou 2003), pasando a una fase de fluencia secundaria o a una fase de fluencia terciaria cuando el nivel de carga es elevado (Bodig y Jayne 1982, Smith et al. 2003). Los materiales viscoelásticos además presentan relajación en el tiem- 
po, que aunque siendo de la misma naturaleza que la fluencia, ésta se manifiesta como una pérdida gradual de tensión debido a una deformación constante en el tiempo (Bodig y Jayne 1982).

El fenómeno de mecanosorción se caracteriza por un cambio en la humedad relativa bajo una carga constante provocando un aumento de la deformación con respecto a una pieza que tiene las mismas limitaciones sin variación de humedad relativa (Husson 2009). La combinación de nivel de carga y de humedad relativa en la respuesta de la fluencia en la madera puede inducir a una ruptura prematura (Hearmon y Paton 1964, Martensson 1988, Liu 1993, Wu y Milota 1996). Por otro lado, la influencia de la temperatura en las propiedades mecánicas de la madera suele evaluarse observando cambios en los parámetros de elasticidad, resistencia, propiedades de fluencia o de relajación de tensiones, o alguna combinación de estos (Bodig y Jayne 1982). La aplicación de elevadas temperaturas en la madera provoca degradaciones químicas más o menos importantes de sus diferentes componentes, induciendo una relajación molecular y un cambio en las propiedades mecánicas (Skaar 1972, Navi y Heger 2005). Varios estudios analizan el efecto de la temperatura en las propiedades mecánicas de la madera. A modo de ejemplo se puede mencionar a Fridley et al. (1989), quienes mostraron que el tiempo de falla del material disminuye con el aumento de la temperatura a una misma carga. Por otro lado, Placet et al. (2008) mostraron que la rigidez de la madera disminuye significativamente a temperaturas superiores a los $80^{\circ} \mathrm{C}$. Del mismo modo, Jouve y Sales (1986) demostraron que al variar la temperatura en un rango entre $20^{\circ} \mathrm{C}$ y $90^{\circ} \mathrm{C}$, la fluencia se hace más importante que cuando se trabaja a temperatura constante de $90^{\circ} \mathrm{C}$.

\section{Uso de preservantes en tableros tensados de madera}

Los preservantes más utilizados en tableros tensados de madera son del tipo oleosolubles, tales como, creosota, pentaclorofenol y naftenato de cobre (Ritter 1990, Wacker et al 2003, DelDOT 2005, Bigelow et al. 2007, Bigelow et al. 2009). Éstos otorgan una buena protección contra la pudrición de estas estructuras, así como también, contra los efectos del clima y condiciones cíclicas de humedad. Este tipo de preservante minimiza el intercambio de agua con el ambiente, dada su naturaleza hidrorepelente, aumentando la estabilidad dimensional de la madera (Andana 2009). Un estudio de monitoreo de puentes de tableros tensados de madera tratados con diversos preservantes, fue llevado a cabo por Kainz et al. 1996, demostrando que aquellos puentes que estuvieron tratados con preservantes oleosolubles retenían mayor fuerza en la barra que los tableros tratados con preservantes hidrosolubles tales como las sales Cobre, Cromo, Arsénico (CCA). Por otro lado, Ranta-Maunus y Kortesmaa (2000) establecieron que la mayor deformación por fluencia se logró en vigas no tratadas; mientras que la menor deformación se produjo en vigas impregnadas con creosota.

En Chile la investigación en esta área ha sido llevada a cabo por las universidades de Concepción y del Bío-Bío a través de proyectos Innova y proyectos Fondef. Las iniciativas de la Universidad del Bío-Bío, se manifiestan en la construcción de la pasarela Mutual en Talcahuano, la cual está diseñada para resistir cargas de tipo peatonal. La Universidad de Concepción en cambio, ha llevado adelante investigaciones sobre puentes postensados de madera que han permitido construir en Chile puentes con esta tecnología y que se encuentran actualmente en uso para resistir cargas del tipo vehiculares, Fondef (2000), Huaquilaf (2004), Corfo-Innova (2008), Andana (2009), Corma (2010), Tapia (2011), Giuliano et al. (2011).

A pesar de estas investigaciones, el estado del arte en el área de tableros tensados, refleja aún escaza información con respecto a las características reológicas de la madera de pino radiata, con y sin tratamiento de preservación, utilizada en este tipo de estructuras en Chile. El objetivo de este estudio fue el de analizar el comportamiento reológico y termo-higromecánico en muestras de tableros de madera tensados de pino radiata, tratados con distintos preservantes, bajo ciclos de humedad relativa y temperatura. Lo anterior permitirá potenciar el estado del arte actual, de manera de mostrar a las cualidades de este material en el uso de construcciones viales. 


\section{MATERIALES Y MÉTODOS}

\section{Materia Prima y obtención de secciones de puentes tensados}

Madera de pino radiata de $40 \mathrm{~mm}$ x $140 \mathrm{~mm}$ x $300 \mathrm{~mm}$, seca al 12\%, previamente clasificada estructuralmente, por una empresa chilena como C24, según la norma EN-519, fue utilizada para el estudio. Con estas piezas de madera se fabricaron 16 secciones de puentes de madera tensada (probetas) cuyas dimensiones finales fueron de $150 \mathrm{~cm}$ x $300 \mathrm{~mm}$ x $650 \mathrm{~mm}$. Cada probeta estaba constituida por 13 piezas de madera, comprimidas a través de placas metálicas, representando de esta forma una sección del tablero del puente, a la que se le incorporó transversalmente una barra de acero, calidad ASTM A722, a través de una perforación de $38.1 \mathrm{~mm}$ de diámetro en el centro de la madera, permitiendo efectuar el tensado de éstas, las que fueron colocadas al interior de una cámara de clima (Figura 2). Cuatro grupos de cuatro probetas de puentes tensados cada uno fueron instalados al interior de una cámara de clima. Éstos componían los tratamientos de preservación estudiados (creosota, LOSP y CCA-cera y probetas testigo, designadas como testigo 1) que se explican más adelante (Figura 1).

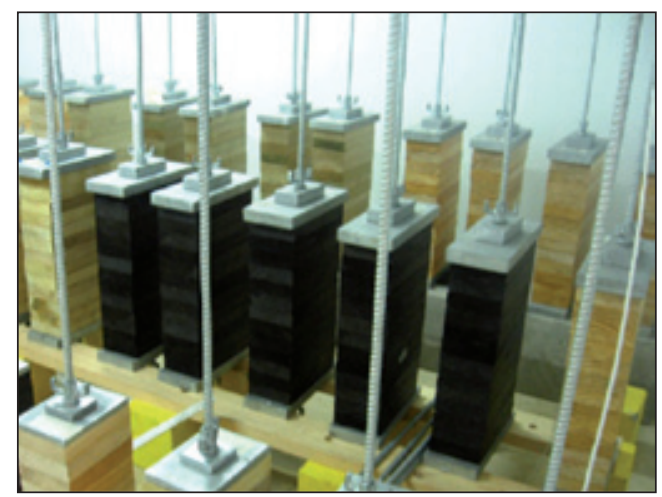

a

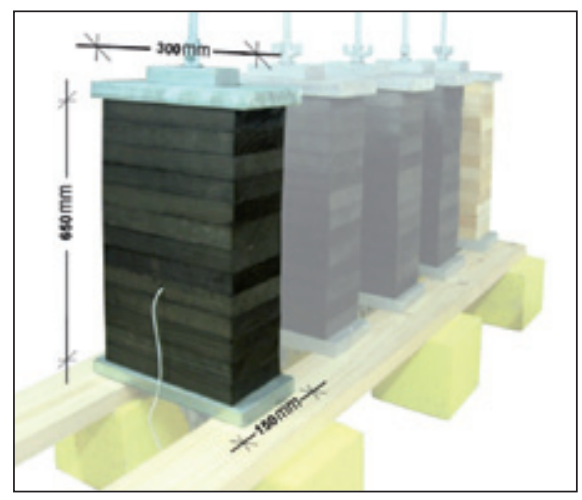

b

Figura 1. Probetas tensadas de madera al interior de una cámara de clima.

a) con y sin tratamiento, b) dimensiones de la probeta.

Después se realizó un tensado de las probetas mediante un cilindro hidráulico, aplicando una carga de $3353 \mathrm{~kg}$ para obtener una tensión interlaminar de $0.78 \mathrm{MPa}$, siguiendo la recomendación para coníferas establecida por Ritter (1990). Después de 7 días en la cámara, se hizo un primer retensado de las barras hasta alcanzar el nivel inicial de tensado (100\%). Luego, después de 28 días en la cámara se realizó un segundo retensado. Lo anterior se efectuó debido a la pérdida de la tensión en las barras sobretodo en las primeras etapas del proceso de tensado. La densidad básica y la densidad de referencia de la madera sin tratamiento de preservación fueron determinadas según la norma chilena NCh 176/2.

\section{Tratamientos de preservación}

Los tratamientos de preservación, con los cuales se trabajó fueron, creosota, LOSP, CCA-cera y probetas testigo 1. La impregnación de la madera se realizó en plantas industriales ubicadas en Yumbel, Coronel y Temuco, respectivamente. En la tabla 1 se muestra la retención promedio obtenida por Tapia (2011) para cada preservante y los valores de retención mínima establecida en la NCh 819. 
Tabla 1. Retenciones promedio y retenciones mínimas de cada tratamiento de preservación.

\begin{tabular}{|c|c|c|}
\hline Tratamiento de Preservación & $\begin{array}{c}\text { Retención Promedio } \\
\left(\mathrm{kg} / \mathrm{m}^{3}\right)^{*}\end{array}$ & $\begin{array}{c}\text { Retención Mínima } \\
\left(\mathrm{kg} / \mathrm{m}^{3}\right)^{* *}\end{array}$ \\
\hline Creosota & 140 & $128-400$ \\
CCA con Cera & 3.91 & 4 \\
LOSP & 0.146 & $0.086-0.26$ \\
\hline
\end{tabular}

*Tapia (2011)

**según NCh 819

\section{Determinación de contenido de humedad}

El contenido de humedad inicial de las probetas tratadas con creosota y LOSP se determinó por el método de destilación, de acuerdo a AWPA A6-01, (American Wood-preservers' Association Standard). Se incluyó además probetas testigos, sin restricción de carga, tratadas con CCA-cera y sin tratar, denominadas éstas últimas como testigo 2, para determinar el contenido de humedad de las piezas por medio del método gravimétrico, según la norma NCh 176/1. Para ambos métodos se utilizaron 5 piezas de madera, sin restricción de carga por cada tratamiento de preservación y un grupo sin tratamiento de preservación, denominadas como testigo 2, todas ubicadas al interior de la cámara de clima para determinar por el método gravimétrico el contenido de humedad según NCh 176/1.

\section{Medición de la tensión interlaminar y deformación de las probetas}

La tensión interlaminar y la deformación de las probetas se determinaron mediante bandas extensométricas del tipo TML modelo PFL-20-11, previamente calibrados. La resistencia de éstos fue de $120 \pm 0.3(\Omega)$, con un coeficiente de expansión térmica de $11.8 \times 10^{-6} /{ }^{\circ} \mathrm{C}$. Se utilizaron dos bandas extensométricas por barra, las que fueron ubicadas en la parte central de éstas y paralelo a su eje longitudinal (Figura 2). Las bandas extensométricas se cubrieron con cinta aislante para prevenir el efecto del roce y lana de vidrio para protegerlos de las variaciones de la temperatura y humedad. Las lecturas fueron efectuadas mediante un sistema de adquisición de datos conectado a un computador, por lo que la tensión interlaminar de las probetas fue obtenida de forma directa.

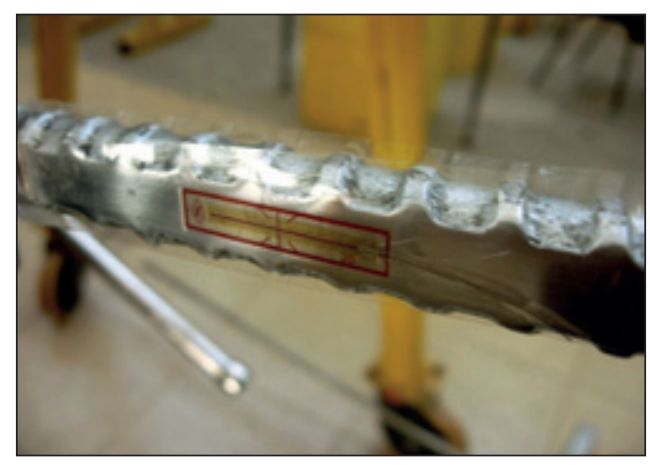

Figura 2. Ubicación de los bandas extensométricas en las barras de tensión.

Por otro lado, a través de un transductor de desplazamiento ubicado en la parte superior de las muestras de puentes tensados y apoyado en la placa metálica y alineado con las barras, se midió directamente la separación entre las placas y por consiguiente la deformación unitaria de las probetas. 


\section{Acondicionamiento en cámara de clima}

Las probetas fueron acondicionadas en una cámara de clima considerando una estación de verano $\left(20^{\circ} \mathrm{C}\right.$ y $\left.65 \% \mathrm{HR}\right)$ y una estación de invierno $\left(5^{\circ} \mathrm{C}\right.$ y $\left.85 \% \mathrm{HR}\right)$. Estas condiciones representan los ambientes estivales medios de ambas estaciones en la undécima región (Coyhaique), zona donde según se emplazarán puentes con tableros tensados de madera de pino radiata, CORMA (2009). Las condiciones de las estaciones se obtuvieron a través de curvas de correlación entre temperatura y humedad relativa tomadas por Gallegos (2009) para la zona comprendida entre Coyhaique y Balmaceda. Para los análisis se consideró que un ciclo correspondía a una estación de verano y a una estación de invierno. Esto implicó suponer que las probetas se sometieron a 1 año en las condiciones de la undécima región. Las probetas estuvieron expuestas a 4 ciclos, equivalente a 4 años de exposición en condiciones ambientales, las cuales fueron condiciones ambientales más exigentes que las reales, ya que no se contempla la exposición a primavera y otoño.

En la figura 3 se muestran las condiciones de temperatura y humedad relativa a los cuales estuvieron expuestas las probetas en función del tiempo. En la primera estación de verano tanto las condiciones de temperatura como de humedad relativa al interior de la cámara de clima se estabilizaron alrededor del día 90. La estación verano del ciclo 2 sufrió los efectos del terremoto que hubo en Chile el 27 de Febrero de 2010, por lo que se tuvo que mantener las probetas al interior de la cámara en esa condición por 60 días aproximadamente.

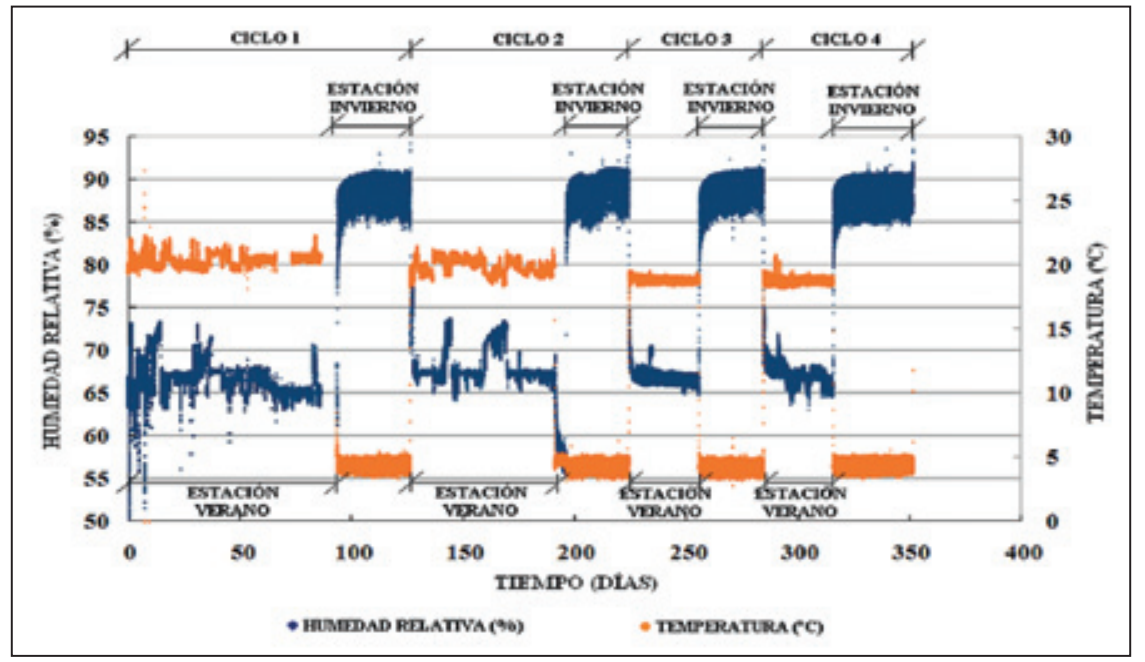

Figura 3. Ciclos de temperatura y humedad relativa al interior de la cámara de clima.

\section{Estabilidad dimensional}

El control del contenido de humedad de las piezas de madera y por consiguiente la estabilidad dimensional de éstas frente a los ciclos de temperatura y humedad, fueron controlados a través de la medición de la contracción e hinchamiento de piezas testigos de madera dejadas al interior de la cámara de clima. Estas piezas, tenían dimensiones similares a las utilizadas en las probetas de tableros tensados, es decir, de $40 \mathrm{~mm}$ x $120 \mathrm{~mm}$ x $140 \mathrm{~mm}$, y sin restricción de carga mecánica. 


\section{Evaluaciones}

La tensión interlaminar y las deformaciones de las probetas se registraron cada media hora en cada ciclo, obteniendo de esta forma, las curvas de deformación unitaria y de pérdida de tensado en el tiempo. La temperatura al interior de la madera se registró mediante termocuplas ubicadas en el centro de cada probeta. Por otro lado, a través de piezas de madera de $40 \mathrm{~mm}$ x $120 \mathrm{~mm}$ x $140 \mathrm{~mm}$, las cuales estaban libres de todo tipo de carga, se determinó la contracción e hinchamiento propio de ellas, a través de medición directa del largo, ancho y espesor, utilizando un vernier. Estas piezas de madera se encontraban al interior de la cámara de clima con el fin que tuvieran las mismas condiciones ambientales que las probetas.

\section{Análisis estadístico}

Un análisis de varianza (ANOVA) de tres factores con medidas repetidas fue aplicado para analizar los resultados, utilizando el programa SAS 9.2. Los factores considerados para este análisis fueron el tipo de tratamiento en 4 niveles (Control, CCA-cera, LOSP y Creosota); el número de ciclos en 4 niveles ( 1 a 4 ) y la estación en 2 niveles (invierno y verano). Los factores repetidos fueron el ciclo y la estación. Se utilizó el procedimiento MIXED del programa SAS con una sentencia repetida y una estructura de covarianza que minimiza el criterio de Akaike. El método de Kenward-Roger fue utilizado para la definición de los grados de libertad y el método LSD de Fisher para el análisis de comparaciones múltiples a un nivel de significancia de $\alpha=0.01$.

Las respuestas analizadas estadísticamente fueron el promedio y la amplitud de la tensión interlaminar para cada probeta en cada estación de cada ciclo. Las mismas respuestas fueron consideradas para el análisis de la deformación de las probetas.

\section{RESULTADOS Y DISCUSIÓN}

\section{Condiciones de la madera en atmósferas climatizadas}

La figura 4 muestra los contenidos de humedad promedio de las probetas al interior de la cámara de clima en función del tiempo para los diferentes tratamientos de preservación. En las muestras testigo (control) se incluyen los valores de contenido de humedad tanto para las piezas de madera de $40 \mathrm{~mm}$ x $120 \mathrm{~mm}$ x $140 \mathrm{~mm}$, utilizadas para la medición de la contracción e hinchamiento, sin carga (testigo 2), así como para las probetas tensadas (testigo 1). Como se puede apreciar, la variación del contenido de humedad entre ambas muestras testigo es aproximadamente $1 \%$ en las estaciones de verano y $2 \%$ en las estaciones de invierno, siendo las piezas de madera sin restricción (testigo 2) las que presentan mayor contenido de humedad. Este resultado era esperable, debido a que las probetas contienen laminaciones de mayor tamaño, además de la restricción que éstas poseen en sentido perpendicular a las fibras. Sin embargo, esta información da una idea aproximada de las variaciones del contenido de humedad en la cámara de clima para todas las probetas, existiendo una diferencia que excede el $2 \%$. De acuerdo a lo anterior, se observa que el contenido de humedad promedio de todas las probetas al ingreso de la cámara fue aproximadamente del $12.5 \%$ y el contenido de humedad promedio al término del ensayo, en la estación invierno del ciclo 4, fue aproximadamente del $15.3 \%$. En general el contenido de humedad promedio de todas las probetas en la estación verano fue aproximadamente del $12.6 \%$ mientras que en la estación invierno del 15.3\%. 


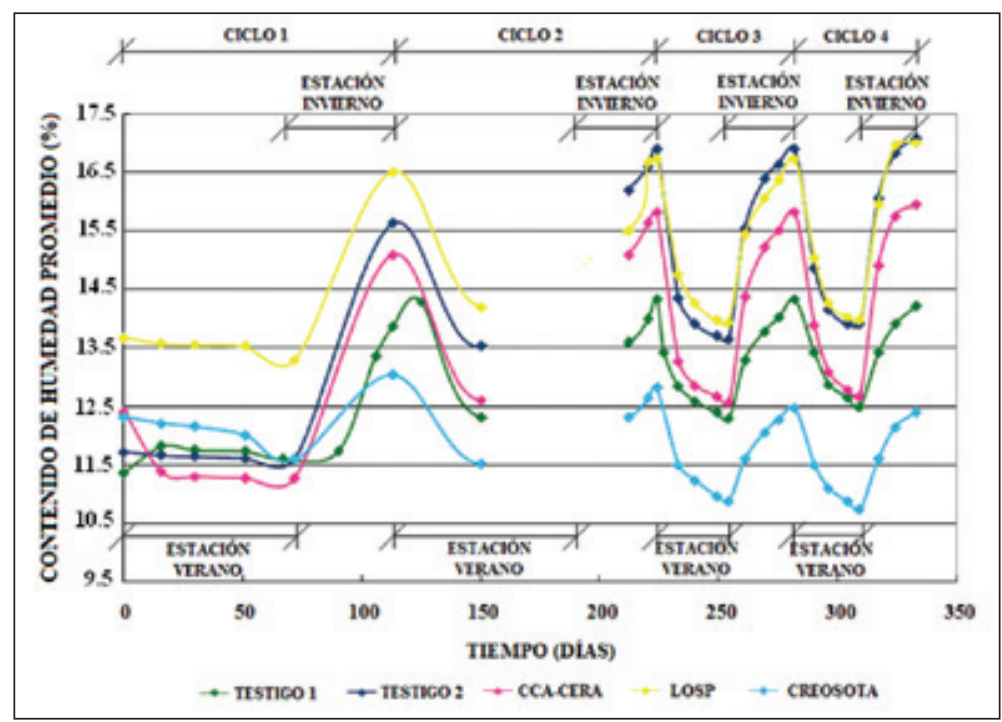

Figura 4. Contenido de humedad controlados al interior de la cámara de clima

a) muestras testigo, b) muestras de madera impregnada.

Con cada condición al interior de la cámara de clima, el contenido de humedad de equilibrio (CHE) esperado en la estación verano $\left(20^{\circ} \mathrm{C}\right.$ y $\left.65 \% \mathrm{HR}\right)$ es de $12 \%$ y para la estación invierno $\left(5^{\circ} \mathrm{C}\right.$ y $85 \% \mathrm{HR}$ ), $18 \%$. De acuerdo a los resultados obtenidos, las probetas no alcanzaron estas humedades, debido al tamaño de las probetas con respecto al tiempo por cada estación.

La temperatura promedio al interior de las probetas fue de aproximadamente $17^{\circ} \mathrm{C}$ en la estación verano, y en la estación invierno alcanzó los $4.5^{\circ} \mathrm{C}$.

La densidad básica y de referencia promedios de las probetas testigo fue de $0.433 \mathrm{~g} / \mathrm{m}^{3}$ y $0.565 \mathrm{~g} /$ $\mathrm{m}^{3}$, respectivamente, las cuales fueron obtenidas antes del ingresar a la cámara de clima.

\section{Tensión Interlaminar}

La figura 5 muestra las tensiones interlaminares promedio medidas mediante bandas extensométricas ubicados al centro de las barras de acero para cada uno de los tratamientos obtenidas durante los 350 días del estudio. Se observa que después de 7 días, las probetas perdieron entre un $25 \%$ y un $40 \%$ del tensado inicial, por lo que se efectuó un retensado de las barras hasta el 100\% (0.78 MPa). Posteriormente, la tensión interlaminar comienza nuevamente a disminuir, sin embargo, esto ocurre a una menor tasa, puesto que a 7 días después del primer retensado, las probetas perdieron entre el $10 \%$ y el $20 \%$ del tensado inicial. Una vez transcurridos 28 días desde el comienzo del ensayo, se realizó un segundo y último retensado, con el fin de disminuir una vez más la pérdida de tensión interlaminar de las probetas en el transcurso del tiempo. Es así como a los 7 días después del segundo retensado, las probetas perdieron alrededor de un 3\% a un 13\% del tensado inicial. Estos resultados concuerdan con lo expuesto por Ritter (1990), quien afirma que la pérdida de tensión en las barras puede ser controlada con el número de veces que el tablero es retensado. 


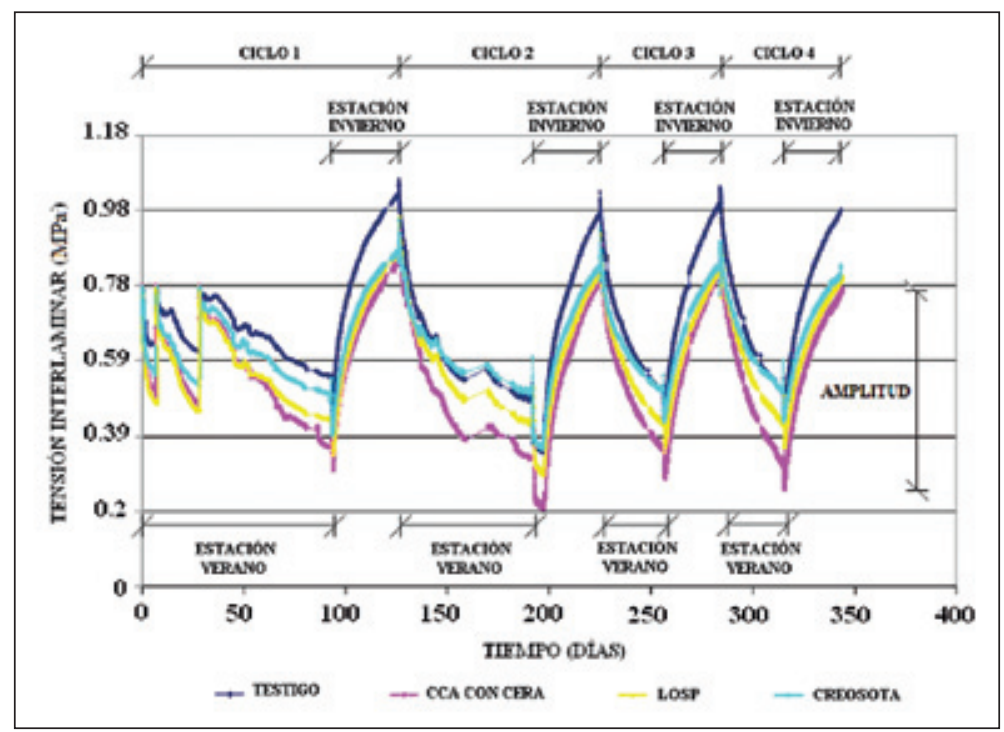

Figura 5. Tensión interlaminar promedio obtenido de 4 probetas de puentes para cada tratamiento de preservación bajo ciclos ambientales en función del tiempo.

En la etapa final de la primera estación de verano, la tensión interlaminar siguió disminuyendo entre un $31 \%$ y $50 \%$ del tensado inicial (Figura 5). Al comenzar la estación de invierno y como era de esperar, la tensión interlaminar comienza a aumentar alcanzando entre un $10 \%$ y un $25 \%$ más del tensado inicial debido a que la madera capta humedad del ambiente. En los ciclos siguientes se observa un comportamiento similar, generándose pérdidas de tensado promedio del $40 \%$ aproximadamente en las estaciones de verano y aumentos de aproximadamente un $20 \%$ en las estaciones de invierno con respecto al tensado inicial. La contracción térmica del acero fue medida, obteniendo una deformación unitaria del acero de $6.337 \times 10^{-6}$, para un diferencial de $15^{\circ} \mathrm{C}$ de temperatura, no influyendo en las variaciones de la tensión interlaminar de las probetas.

Dos fenómenos pudieron haberse producido para las variaciones encontradas en la tensión interlaminar de los diferentes ciclos. La fuerza de la barra pudo verse alterada con el tiempo, debido a un posible fenómeno de relajación de la madera, dado que ésta se encuentra comprimida perpendicularmente. Por otro lado, los esfuerzos en las barras también pudieron verse afectados debido a cambios dimensionales de las piezas de madera que conforman las probetas, dado que los contenidos de humedad de éstas se encuentran por debajo del punto de saturación de las fibras (PSF). Es importante observar que la tensión promedio permanece constante obteniéndose una pérdida de tensado de un $20 \%$ en todos los ciclos, con valores máximos y mínimos contantes.

La figura 5 también muestra una disminución acelerada de la tensión interlaminar al inicio de la estación invierno y un aumento acelerado al inicio de la estación verano de las probetas, esto podría asociarse a la disminución y al aumento de la temperatura, respectivamente por el cambio de estación; sin embargo, este rápido efecto no prevalece en el tiempo.

\section{Análisis Estadístico}

\section{Promedio y Amplitud de la Tensión Interlaminar}

La tabla 2 muestra el análisis de varianza (ANOVA) realizado al promedio y a la amplitud de la tensión interlaminar $(\alpha=0.01)$. La tensión interlaminar mostró interacciones de segundo orden entre 
Ciclo Estación, Tratamiento ${ }^{\star}$ Estación y Tratamiento ${ }^{\star}$ Ciclo, indicando un efecto adicional debido a la influencia combinada de cada uno de estos dos factores.

Tabla 2. Análisis de varianza del promedio y amplitud de la tensión interlaminar. Test de efectos fijos.

\begin{tabular}{lccccc}
\hline & \multicolumn{2}{c}{ PROMEDIO } & \multicolumn{2}{c}{ AMPLITUD } \\
\hline \multicolumn{1}{c}{ EFECTO } & ${ }^{*}$ DF & Valor F & ${ }^{*}$ Pr $>$ F & Valor F & Pr $>$ F \\
Tratamiento & 3 & 19.92 & 0.0015 & 71.35 & $<.0001$ \\
Ciclo & 3 & 56.60 & $<.0001$ & 33.75 & $<.0001$ \\
Tratamiento ${ }^{*}$ Ciclo & 9 & 3.39 & 0.0024 & 9.51 & $<.0001$ \\
Estación & 1 & 1152.29 & $<.0001$ & 263.21 & $<.0001$ \\
Tratamiento ${ }^{*}$ Estación & 3 & 58.64 & $<.0001$ & 5.73 & 0.0013 \\
Ciclo $^{*}$ Estación & 3 & 60.48 & $<.0001$ & 67.18 & $<.0001$ \\
Tratamiento ${ }^{*}$ Ciclo ${ }^{*}$ Estación & 9 & 0.83 & 0.5899 & 2.75 & 0.0095 \\
\hline
\end{tabular}

${ }^{\star}$ DF: Grados de libertad ${ }^{*}$ Pr: Valor P

Los resultados de este análisis mostraron por ejemplo, una disminución significativa de la tensión interlaminar en la estación verano y un aumento significativo en la estación invierno para todas las probetas (Figura 5).

Durante el primer ciclo, tanto en verano como en invierno, se observan mayores valores de tensión, con respecto a los ciclos siguientes. Este efecto se debió principalmente al efecto de compresión que provoca en la probeta la actividad de retensado en el sistema. Por otro lado, no hubo diferencias significativas entre las probetas tratadas con creosota y las probetas testigo 1 en este mismo ciclo. Las probetas tratadas con CCA-cera y LOSP, obtuvieron valores de tensión laminar más bajos en la estación de verano, comparadas con las otras probetas en todos los ciclos. Durante la estación invierno, las probetas testigo 1 presentaron diferencias significativas en el promedio de la tensión interlaminar con respecto a las probetas tratadas. En general, los preservantes de madera hacen cambiar los mecanismos de absorción de agua en la madera tratada (Figura 5). Sin embargo, no hubo diferencias significativas en la tensión interlaminar entre las probetas tratadas con preservantes químicos.

En los ciclos 3 y 4 no se evidenciaron diferencias significativas en el promedio de la tensión interlaminar de todas las probetas, por lo que se descartó evidencia de relajación de la tensión, (Figura 5). Lo anterior podría atribuirse a un bajo nivel de carga aplicado inicialmente en las probetas. Esto concuerda con los estudios de Hearmon y Paton (1964), y por Martensson (1988), quienes demostraron la importancia del nivel de carga en la respuesta de la fluencia en la madera.

Por otro lado, el ANOVA aplicado a la amplitud de la tensión interlaminar mostró interacción triple en Tratamiento ${ }^{\star} \mathrm{Ciclo}^{\star}$ Estación, lo que hace el análisis complejo, dado los efectos puede ser causados por la significación de cualesquiera de las siguientes interacciones: Tratamiento por Ciclo x Estación, o Ciclo por tratamiento x Estación, Estación por tratamiento x Ciclo. De este modo, los resultados mostraron que las probetas tratadas con CCA-cera se comportaron de manera similar que las probetas testigo 1 y que la amplitud es similar en todas las estaciones de todos los ciclos, excepto en la estación verano del ciclo 1. En general, las probetas con CCA-cera presentaron menor estabilidad dimensional en comparación con los otros tratamientos de preservación, lo que puede deberse a la baja cantidad de cera utilizada o a la heterogeneidad de la emulsión CCA-cera, reforzándose lo establecido por Ritter en 1990 sobre los preservantes hidrosolubles. Las probetas tratadas con creosota 
y LOSP presentaron amplitudes similares en todas las estaciones de todos los ciclos, a excepción de la estación verano en el ciclo 1, producto del retensado. Según Andana (2009), las maderas tratadas con creosota presentan mejor estabilidad dimensional que las tratadas con preservantes hidrosolubles y que aquellas sin tratamiento. Finalmente, entre los ciclos 3 y 4 y las estaciones verano e invierno, las amplitudes de tensión interlaminar de todas las probetas no presentan diferencias significativas, indicando que las variaciones dimensionales de contracción e hinchamiento fueron similares ante los cambios ambientales en el tiempo.

\section{Deformación unitaria}

La figura 6 muestra las deformaciones unitarias promedio producidas en las probetas con y sin tratamiento de preservación. Estas deformaciones fueron consideradas positivas en la dirección de la carga y viceversa. De esta manera, las contracciones de las probetas fueron registradas con signo positivo, mientras que los hinchamientos, con signo negativo. Estas deformaciones podrían haberse producido por la compresión perpendicular a las fibras, pues la deformación de las células de la madera afecta a la contracción o al hinchamiento de la madera. Esto concuerda con Skaar (1972), quien mencionó que las tensiones mecánicas (tensión o compresión) pueden causar una deformación permanente de las células de la madera, afectando a su vez a la contracción e hinchamiento.

Los resultados muestran un comportamiento similar en la deformación tanto para las probetas impregnadas como para las testigos. La tasa de deformación es alta principalmente hasta el primer retensado, disminuyendo entre el primer y segundo retensado. Después del segundo y último retensado, la tasa de deformación disminuyó paulatinamente, sin llegar a estabilizarse. Al término de la estación verano en el ciclo 1 , la deformación alcanzó valores de $0.17 \%$, para las probetas testigo, y de $0.32 \%$, para las probetas tratadas con CCA-cera y LOSP. Al inicio de la estación de invierno del ciclo 1 , la deformación disminuyó, debido a que la madera se hincha con el aumento de la humedad relativa, alcanzando valores entre el $0.08 \%$, para la probeta testigo, y $0.23 \%$ para las probetas tratadas con CCA-cera y LOSP.

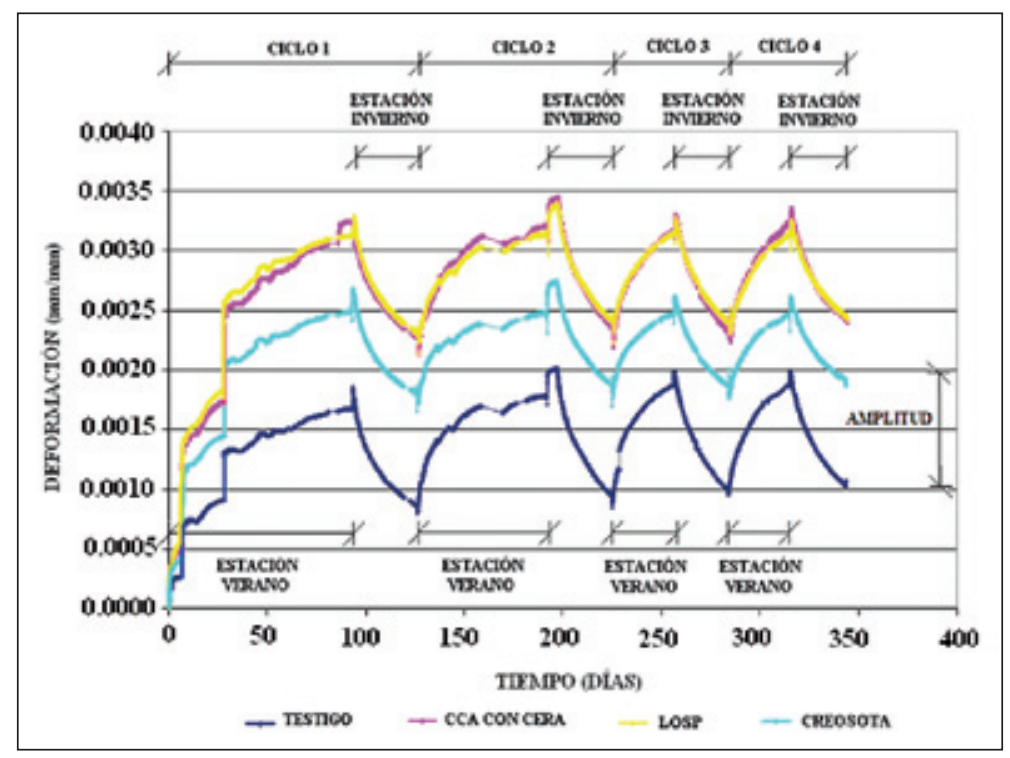

Figura 6. Deformación unitaria promedio obtenida de 4 probetas de puentes para cada tratamiento de preservación bajo ciclos ambientales en función del tiempo. 
Al igual que la tensión interlaminar, no se observó un aumento en la deformación a través de los ciclos en todas las probetas. Sin embargo, si hubo un aumento acelerado de la deformación al inicio de la estación invierno y una disminución acelerada al inicio de la estación verano. Esto podría asociarse a la disminución y al aumento brusco de la temperatura que se produce en los cambios de ciclo; sin embargo, este efecto no prevalece en el tiempo.

\section{Análisis Estadístico}

\section{Promedio y Amplitud de la Deformación}

La tabla 3 muestra que el análisis de Varianza $(\alpha=0.01)$, aplicado a la deformación, tanto para el promedio como para la amplitud de ésta. Los resultados mostraron interacciones de tercer orden en Tratamiento ${ }^{\star}$ Ciclo ${ }^{\star}$ Estación. La deformación promedio presentó diferencias significativas entre la estación de verano del ciclo 1 y el resto de las estaciones de verano en todas las probetas, debido principalmente al retensado.

Tabla 3. Análisis de Variancia del promedio y amplitud de la deformación. Test de efectos fijos.

\begin{tabular}{cccccc}
\hline & & \multicolumn{2}{c}{ PROMEDIO } & \multicolumn{2}{c}{ AMPLITUD } \\
\hline EFECTO & ${ }^{*}$ DF & Valor F & ${ }^{*}$ Pr $>$ F & Valor F & Pr $>$ F \\
Tratamiento & 3 & 17.02 & 0.0081 & 23.73 & $<.0001$ \\
Ciclo & 3 & 521.27 & $<.0001$ & 196.66 & $<.0001$ \\
Tratamiento ${ }^{\star}$ Ciclo & 9 & 13.22 & $<.0001$ & 8.69 & $<.0001$ \\
Estación & 1 & 0.45 & 0.5080 & 199.83 & $<.0001$ \\
Tratamiento ${ }^{\star}$ Estación & 3 & 74.22 & $<.0001$ & 14.50 & $<.0001$ \\
Ciclo $^{\star}$ Estación & 3 & 254.27 & $<.0001$ & 157.48 & $<.0001$ \\
Tratamiento ${ }^{\star}$ Ciclo ${ }^{\star}$ Estación & 9 & 13.88 & $<.0001$ & 5.80 & $<.0001$ \\
\hline
\end{tabular}

${ }^{\star}$ DF: Grados de libertad ${ }^{*} \operatorname{Pr}$ : Valor P

Por otro lado, los resultados de la deformación promedio mostraron que para cada ciclo en cada estación, las probetas testigos se deformaron menos que las probetas tratadas. Además, los ciclos 3 y 4 no presentaron diferencias significativas en el promedio de la deformación de todas las probetas, por lo que no se evidencia presencia de fluencia termo-higromecánica en este estudio (Figura 7), confirmando lo estudiado por Hearmon y Paton (1964), Martensson (1988).

Los resultados del análisis de la amplitud de la deformación fueron equivalentes a los de la tensión interlaminar, mostrando que las probetas tratadas con CCA-cera presentaron menor estabilidad dimensional y que las probetas tratadas con creosota y LOSP presentaron mayor estabilidad dimensional que las tratadas con preservantes hidrosolubles y que el testigo. Además, entre los ciclos 3 y 4 en las estaciones verano e invierno, las amplitudes de la deformación de todas las probetas fueron similares, no presentaron diferencias significativas, lo que indica que todas las probetas presentan valores constantes de contracción e hinchamiento.

Del análisis anterior, se desprende que tanto la tensión interlaminar como la deformación en los ciclos 3 y 4 se comportaron de manera similar, no existiendo diferencias significativas entre ellos. Esto pone en evidencia que no se manifestó un efecto reológico ya sea de relajación de la tensión ni fluencia termo-higromecánica en ninguna de las probetas. Además, las variaciones de humedad 
relativa influyen tanto en la tensión interlaminar como en la deformación, dado que un mínimo incremento o disminución en el contenido de humedad podría producir un aumento o pérdida de la tensión interlaminar, respectivamente. Sin embargo, a pesar de que las probetas no llegaron al CHE durante las estaciones de verano e invierno, es probable que si haya sido alcanzado superficialmente y no en el centro de éstas, por lo que esta variación superficial del contenido de humedad podría incidir tanto en la tensión interlaminar como en la deformación.

\section{Estabilidad Dimensional}

En la figura 7 se muestran los valores promedio de contracción e hinchamiento radial de todas las probetas a través del tiempo. Los valores de contracción e hinchamiento radial influyen directamente en la tensión y deformación de las probetas de tableros tensados de madera, puesto que ésta es la dirección de la carga aplicada. El hinchamiento radial en las probetas testigo alcanzó valores del 1\% en la estación invierno. Los menores valores de hinchamiento se presentaron en las probetas tratadas con creosota, alcanzando valores del $0.5 \%$ en invierno. Los mayores valores de contracción radial se observaron en las probetas testigo, alcanzando $0.3 \%$; mientras que en las probetas tratadas con creosota se obtuvieron los menores valores, alcanzando $-0.2 \%$.

Como era de esperar, las probetas tratadas con creosota presentaron mayor estabilidad dimensional, observándose menor diferencia entre el hinchamiento y la contracción en cada estación con respecto al resto de las probetas, lo que concuerda con el análisis de amplitud realizado tanto en la tensión interlaminar como en la deformación, coincidiendo también, con lo establecido por Andana (2009) y Bigelow et al. (2009).

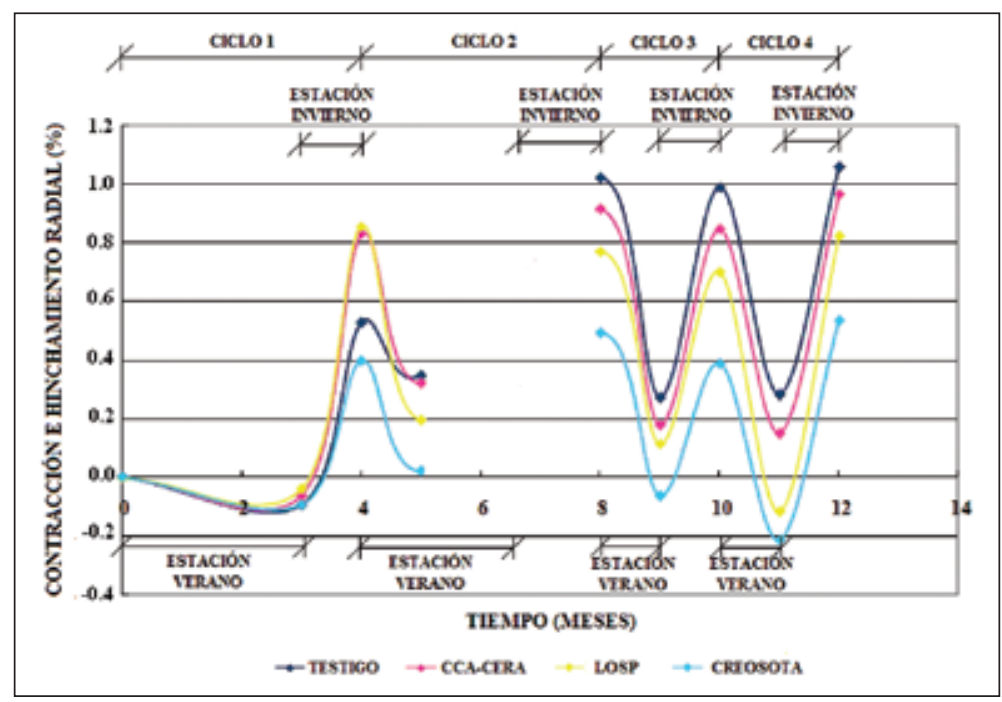

Figura 7. Contracción e Hinchamiento Radial en cada pieza de madera en función del tiempo.

\section{CONCLUSIONES}

En esta investigación se estudió el efecto de variaciones cíclicas de temperatura y humedad relativa en el comportamiento reológico y termo-higromecánico de probetas de tableros tensados de madera de pino radiata, así como también el efecto de los preservantes de madera creosota, CCA-cera y LOSP. En general, se comprobó que la variación de la temperatura y de la humedad tiene una fuerte influencia en la variación de la tensión de las barras tensoras. La pérdida del tensado es considerablemente mayor en el primer período de tiempo, sin embargo, luego de los retensados de las probetas de 
tableros de madera, se minimiza esta pérdida hasta casi estabilizarse. La tensión interlaminar de las probetas de puentes tensados disminuyó significativamente en verano, mientras que la deformación de éstos aumentó. Sin embargo, en la estación invierno, sucedió todo lo contrario. Las variaciones de temperatura afectaron la tensión interlaminar y la deformación al comienzo de cada estación, sin embargo, el cambio de la humedad relativa fue el factor de mayor influencia en cada estación. No se evidenció relajación de la tensión ni fluencia termo-higromecánica para ninguna de las probetas en ninguna de las condiciones ambientales estudiadas para un nivel de tensión interlaminar de 0.78 $\mathrm{MPa}$. Los tableros tensados de madera tratados con creosota y LOSP presentaron mayor estabilidad dimensional que los tratados con CCA-cera y las probetas testigo, lo que influyó directamente en la tensión interlaminar y en la deformación.

\section{AGRADECIMIENTOS}

Los autores agradecen el financiamiento otorgado por el proyecto Corfo Innova "Desarrollo de bases para la implementación de alternativas de construcción y reparación de puentes secundarios, ocupando materias primas ecológicas y sustentables". Al mismo tiempo, agradecen al personal del Laboratorio de Estructuras del Depto. de Ingeniería Civil de la Universidad de Concepción y del Pabellón de Tecnología de la Madera de la Universidad del Bío-Bío por su apoyo logístico durante el desarrollo experimental de este estudio. Además los autores agradecen la beca "Programme des futurs leaders dans les Amériques (PPLA)" que permitió realizar una estadía de investigación en la Université Laval de Canadá y así complementar parte del proyecto.

\section{BIBLIOGRAFÍA}

Andana, F. 2009. Influencia del tratamiento de preservación en tableros de madera tensada de Pinus radiata. Memoria de título. Departamento de Ingeniería Civil, Universidad de Concepción, Concepción, Chile. 182 pp.

ASTM. American Society for Testing and Materials. 2007. Especificación Normalizada para Barras de Acero de Alta Resistencia sin Recubrimiento para Concreto Preesforzado. ASTM A722/ A722M-2007.

AWPA. American Wood Protection Association. 2001. Metohd for the Determination of OilType Preservatives and Water in Wood. AWPA A6-01. 8pp. American Wood Protection Association (formerly the American Wood-Preservers' Association)

Bigelow, J.; Clausen, C; Lebow, S.; Greimann, L. 2007. Field evaluation of Timber preservation treatments for highway applications. Final Report. Bridge Engineering Center. Center for Transportation Research and Education. Iowa State University. USA.106 pp.

Bigelow, J.; Lebow, S.; Clausen, C; Greimann, L.; Wipf, T. 2009. Preservation Treatment for Wood Bridge Application. Transportation Research Record: Journal of the Transportation Research Borrad. 2108: 77-85.

Bodig, J.; Jayne, B.A. 1982. Mechanics of Wood and Wood Composites. Van Nostrand Reinhold. New York. 712 pp.

Bou, S.E. 2003. Contribution a la modélisation des effects différés du bois et du beton sous conditions climatiques variables. Aplication aux structures mixtes bois-beton. Tesis de grado de Doctor. L' Institut National des Sciences Appliquées de Lyon. École doctorale des Sciences de L' ingénieur de 
Lyon: Mécanique, Energétique, Génie civil, Acoustique. France. 174 pp.

CORFO-INNOVA. 2008. Desarrollo de Bases para la Implementación de Alternativas de construcción y Reparación de Puentes Secundarios, ocupando Materias Primas Ecológicas y Sustentables. Código Proyecto: 07CT7ICM-16. Chile.

CORMA, Corporación Chilena de la Madera. 2009. Puentes tensados de madera una iniciativa desde la región del Bío-Bío. Boletín electrónico Forestal. Corma Bío-Bío. [en línea]. Disponible en $<$ http://www.cormabiobio.cl/boletin/noticiadestv2.php? codigo=925 $>$, [consultado 12 de Noviembre 2011].

CORMA, Corporación Chilena de la Madera. 2010. Con éxito operan en Chile los puentes

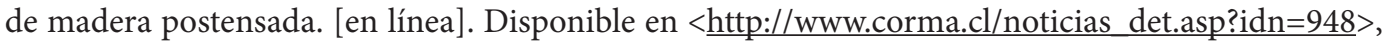
[consultado 20 de Diciembre 2011].

DelDOT. 2005. DelDOT Bridge Design Manual. Chapter eight. Timber structures Design. Department of transportation, The quality section. State of Delaware. $387 \mathrm{pp}$.

Dyken, T. 2005. Monitoring of Norwegian timber bridges. Proceedings NVF Timber Bridges. Nordic roads Association, Norwegian Public Roads Administration. Hamar. 13 pp.

EN 519. 1995. Structural timber. Grading. Requirements for machine strength graded timber and grading machines.

FONDEF. 2000. Protección por diseño en el uso de madera en la infraestructura vial: puentes, pasarelas y elementos de seguridad. Proyecto Fondef - Conicyt: D00i1164. [en línea]. Disponible en $<$ www.fondef.cl/bases/fondef/PROYECTO/00/I/D00I1164.HTML $>$ [consultado Diciembre 2011].

Fridley, K.; Tang, R. C.; Soltis, L. 1989. Thermal effects on load-duration behavior of lumber. Part 1. Effect of constant temperature. Wood and Fiber Sci. 21(4): 420-431.

Gilún, A.; Meronk, J. 2006. Stress-laminated timber T-beam and box-beam bridges. Tesis de Magister. International Master's programme in Structural Engineering. Department of Civil and Environmental Engineering. Division of Structural Engineering. Steel and Timber Structures. Chalmers University of Technology. Göteborg, Suecia. 141 pp.

Giuliano, M.P.; Dechent- Silva, R. 2011. Manual de diseño, construcción, mantención y monitoreo de tableros de madera tensado. Universidad de Concepción, Facultad de Ingeniería Civil, Departamento de Ingeniería Civil, Concepción, Chile. 157 pp.

Gallegos, B. 2009. Influencia de la Temperatura y Humedad en Tableros para Puentes de Madera Tensados. Memoria de título. Departamento de Ingeniería Civil, Universidad de Concepción, Concepción, Chile. 137 pp.

Hearmon, R. F. S.; Paton, J.M. 1964. Moisture content changes and creep of wood. For. Prod. J. 14: 357-359.

Huaiquilaf, B. 2004. Estudio Experimental del Comportamiento Reológico de Tableros Tensados de Madera Pino Radiata Endurecida (PRE). Memoria de título, Departamento de Ingeniería Civil, Universidad de Concepción, Concepción, Chile. 138 pp. 
Husson, J.M. 2009. Loi de comportement viscoélastique avec effect mémoire. Application à la mecanosorption dans le bois. Tesis de grado de Doctor. Faculté des Sciences et Techniques, Université de Limoges.120 pp.

NCh176/1.1986. Maderas. Determinación de la humedad. Instituto de Normalización Nacional, Santiago.

NCh176/2.1986. Maderas. Determinación de la densidad. Instituto de Normalización Nacional, Santiago.

NCh819. 2009. Madera Preservada-Pino Radiata-Clasificación Según Uso y Riesgo en Servicio y Muestreo. Instituto de Normalización Nacional, Santiago. Chile. Documento interno en proceso.

Jouve, J. H.; Sales, C. 1986. Influence de traitements physic-chimiques sur le fluage du matériau bois et la relaxation des contraintes. Séminaire interne 22-24 sept. (Nogent/Vernisson) GS Rhéologie du bois CNRS, France.

Kainz, J.; Ritter, M.; Yazdani, N.; Kadnar, J. 1996. Effect of preservative treatment on bar force in stress-laminated bridge decks. In: Ritter, M.; Duwadi, S.; Lee, P., eds. National conference on wood transportation structures; Madison, WI. Madison, WI: U.S. Department of Agriculture, Forest Service, Forest Products Laboratory. Gen. Tech. Rep. FPL-GTR-94: 245-251.

Kainz, J.; Wacker, J.; Ritter, M. 2001. Analysis of thermal change in stress laminated timber bridge decks. Madison, WI: U.S. Department of Agriculture, Forest Service, Forest Products Laboratory. Res. Pap. FPL.RP.598. 9 pp.

Liu, T. 1993. Creep of wood under a large span of loads in constant and varying environments. Part 1: Experimental observations and analysis. Holz als Roh und Werkstoff 51: 400-405.

Martensson, A. 1988. Tensile behaviour of hardboard under combined mechanical and moisture loading. Wood Sci. Technol. 22:129-142.

Navi, P.; Heger, F. 2005. Comportement thermo-hygromécanique du bois. Presses Polytechniques et Universitaires Romandes, Suisse-Lausanne.

Placet, V.; Passard, J.; Perré, P. 2008. Viscoelastic properties of wood across the grain measured under water-satured conditions up to $135^{\circ} \mathrm{C}$ : evidence of thermal degradation. J. Mater Sci. 43:32103217.

Ranta-Maunus, A.; Kortesmaa, M. 2000. Creep of timber during eight years in natural environments. World Conference on Timber Engineering. Canada.

Ritter, M. 1990. Timber Bridges: Design, Construction, Inspection and Maintenance. U.S. Department of Agricultura, Forest service, Forest Products Laboratory, Madison, Wisconsin.

Ritter, M. A.; Lee, P. D. H.; Porter, G. J. 1996. Field performance of timber bridges: 6. Hoffman Run stress-laminated deck bridge. Madison, WI: U.S. Department of Agriculture, Forest Service, Forest Products Laboratory, Res. Pap. FPL.RP.549.16 pp.

SAS Institute Inc. 2008. SAS 9.2 Software. Cary, NC. USA.

Skaar, C. 1972. Water in wood. N.Y. Syracuse. USA. University Press. 218 pp. 
Smith, I.; Landis, E.; Gong, M. 2003. Fracture and fatigue in wood. John Wiley and Sons Ltd. England.

Tapia, O. 2011. Tratamiento en Tableros de Pino Radiata Destinados a la Construcción de Puentes de Madera Tensado. Memoria de título, Departamento de Ingeniería Civil, Universidad de Concepción, Chile. 166 pp.

Ugalde, G. 2004. Diseño y Construcción Tablero de Madera Tensado para el Puente Cautín. Memoria de título, Departamento de Ingeniería Civil, Universidad de Concepción, Concepción, Chile. $183 \mathrm{pp}$.

Wacker, J.P.; Crawford, D.M.; Erickson, M. 2003. Creoste retension levels of timber highway bridge superstructures in Michigan's lower peninsula. Forest Service. Forest Products Laboratory. Research note FPL-RN-0289. 17 pp.

Wacker, J.P.; Seavey, R.; Erickson, R. 1996. Cold Temperature Effects on Stress-Laminated Timber Bridges. In: Ritter, M.A.; Duwadi, S.R.; Lee, P.D.H., ed(s). National conference on wood transportation structures. October 23-25, 1996. Madison, WI: U.S. Gen. Tech. Rep. FPL- GTR-94. Department of Agriculture, Forest Service, Forest Products Laboratory.

Wu, Q.; Milota, M. 1996. Mechano-sorptive deformation of douglas-fir specimens under tangential tensile stress during moisture adsorption. Wood and Fiber Sci. 28(1):128-132. 
